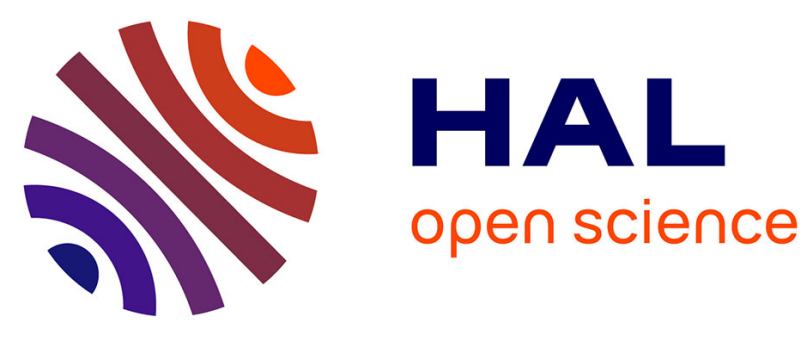

\title{
Source Separation of Baseband Signals in Post-Nonlinear Mixtures
}

\author{
Leonardo Tomazeli Duarte, Christian Jutten, Bertrand Rivet, Ricardo
}

Suyama, Romis Attux, João Marcos Romano

\section{- To cite this version:}

Leonardo Tomazeli Duarte, Christian Jutten, Bertrand Rivet, Ricardo Suyama, Romis Attux, et al.. Source Separation of Baseband Signals in Post-Nonlinear Mixtures. MLSP 2009 - IEEE 19th International Workshop on Machine Learning for Signal Processing, Sep 2009, Grenoble, France. pp.in CD. hal-00416161

\section{HAL Id: hal-00416161 \\ https://hal.science/hal-00416161}

Submitted on 12 Sep 2009

HAL is a multi-disciplinary open access archive for the deposit and dissemination of scientific research documents, whether they are published or not. The documents may come from teaching and research institutions in France or abroad, or from public or private research centers.
L'archive ouverte pluridisciplinaire HAL, est destinée au dépôt et à la diffusion de documents scientifiques de niveau recherche, publiés ou non, émanant des établissements d'enseignement et de recherche français ou étrangers, des laboratoires publics ou privés. 


\section{SOURCE SEPARATION OF BASEBAND SIGNALS IN POST-NONLINEAR MIXTURES}

\author{
L. T. Duarte*, C. Jutten ${ }^{\dagger}$, B. Rivet \\ GIPSA-lab (UMR CNRS 5216) \\ Institut Polytechnique de Grenoble \\ BP 46 - 38402 Saint Martin d'Hères, France
}

\author{
R. Suyama, R. Attux and J. M. T. Romano \\ School of Electrical and Computer Engineering \\ University of Campinas - UNICAMP \\ 13083-970, CP 6101, Campinas, SP, Brazil
}

\begin{abstract}
Usually, source separation in Post-Nonlinear (PNL) models is achieved via one-stage methods, i.e. the two parts (linear and nonlinear) of a PNL model are dealt with at the same time. However, recent works have shown that the development of two-stage techniques may simplify the problem. Indeed, if the nonlinear stage can be compensated separately, then, in a second moment, one can make use of the well-established source separation algorithms for the linear case. Motivated by that, we propose in this work a novel two-stage PNL method relying on the assumption that the sources are bandlimited signals. In the development of our method, special care is taken in order to make it as robust as possible to noise. Simulation results attest the effectiveness of the proposal.
\end{abstract}

\section{INTRODUCTION}

The objective of blind source separation (BSS) methods is to estimate an unknown set of source signals by using only samples that are mixtures of these original signals. This problem has been extensively studied for the case in which the mixing process is modeled as a linear system. In such situation, the independent component analysis (ICA) is the standard tool to separate the sources. However, when the mixing process is nonlinear, BSS becomes a more complex task. For instance, nonlinear models are, as a rule, not separable. Put differently, the recovery of the condition of statistical independence, which is the very essence of ICA, does not always ensure proper source separation in nonlinear models [1]. In view of this problem, a more reasonable approach in nonlinear BSS is to focus on constrained systems, for which the sound separability properties still hold.

The most studied examples of separable nonlinear models belong to the class of Post-Nonlinear (PNL) models [2]. In PNL models, the sources firstly undergo a linear mixing process. Then, the resulting outputs are submitted to a second stage composed of component-wise nonlinear functions. The usual approach to recover the sources in a PNL model

\footnotetext{
* L.T. Duarte is grateful to the CNPq (Brazil) for funding his $\mathrm{PhD}$ research.

${ }^{\dagger} \mathrm{C}$. Jutten is with the Institut Universitaire de France.

$\ddagger$ Thanks to FAPESP for the financial support.
}

tries to deal with these both stages at the same time by minimizing the mutual information between the outputs of the separating system [2]. Despite the theoretical soundness of such an approach, there are several drawbacks regarding its applicability. For example, the evaluation of the mutual information demands estimation of marginal entropies, which results in a complex task. Also, given the difficulty in defining a MISO (multiple-input single-output) contrast based on the mutual information, it would not be possible to perform source extraction in such a case.

In order to overcome practical problems like these, alternative methods, like the gaussianization [3] and the geometrical [4] approaches, rely on additional assumptions about the sources in order to obtain a two-stage solution, in which the nonlinear and linear stages of the PNL model are treated separately. Indeed, if the nonlinear effects introduced in a PNL model are counterbalanced in a first stage, then the remaining task becomes essentially of linear nature and, thus, can be accomplished in a very efficient way by linear source separation or source extraction methods. Yet, despite the encouraging results provided by the two-stage PNL methods presently available, there are still some limitations that make their application difficult in a practical scenario. For instance, the geometrical method [4] is restricted to scenarios with only two sources, whereas the gaussianization approach [3] may fail when there is a small number of sources.

We propose in this work a novel two-stage PNL method that employs a certain degree of prior knowledge about the spectral content of the sources. More specifically, we assume that the signals of interest are bandlimited, which allows us to formulate a simple yet solid criterion for the estimation of the PNL nonlinear stage. The core of our idea is based on a well-known result from the nonlinear signal processing theory $[5,6]$, namely: when a signal is submitted to a nonlinear function, the spectrum of the resulting signal becomes wider. Therefore, a natural approach to counterbalance nonlinear distortions is to search for a function that gives again a bandlimited signal. We shall show that the application of this result gives a simple PNL source separation method.

In order to present the details of our method, the paper is divided as follows. Firstly, in Section 2, we review the PNL 
mixing model. Then, in Section 3 we describe the main aspects of our approach. In Section 4, we perform a set of simulations with the aim of assessing the validity of our method. Finally, the concluding remarks are present in Section 5.

\section{POST-NONLINEAR MIXING MODEL}

The PNL model, depicted in Fig. 1, consists of a linear mixing stage followed by a set of invertible nonlinearities, each one applied to a single linearly mixed signal. In mathematical terms, the vector containing the mixed signals in a PNL model can be expressed as

$$
\begin{aligned}
\mathbf{x}(t) & =\mathbf{f}(\mathbf{A} \mathbf{s}(t)) \\
& =\mathbf{f}(\mathbf{z}(t))
\end{aligned}
$$

where $\mathbf{s}(t)=\left[s_{1}(t), s_{2}(t), \ldots, s_{n}(t)\right]^{T}$ is the vector containing the source signals and $\mathbf{f}(\cdot)=\left[f_{1}(\cdot), f_{2}(\cdot), \ldots, f_{n}(\cdot)\right]^{T}$ denotes the nonlinearities applied to each output of the linear mixing stage, described by the matrix $\mathbf{A}$.

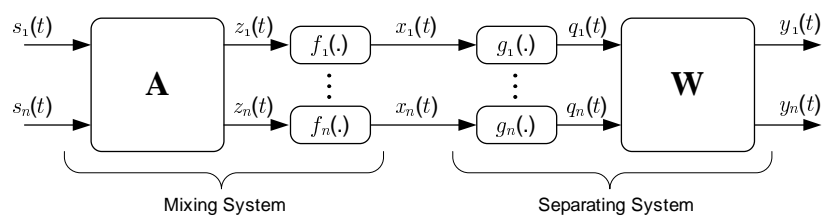

Fig. 1. The PNL problem structure.

Still in Fig. 1, we show the PNL separating system, which is basically a mirrored version of the mixing system. The estimated sources are thus given by

$$
\begin{aligned}
\mathbf{y}(t) & =\mathbf{W g}(\mathbf{x}(t)) \\
& =\mathbf{W} \mathbf{q}(t)
\end{aligned}
$$

where $\mathbf{g}(\cdot)=\left[g_{1}(\cdot), g_{2}(\cdot), \ldots, g_{n}(\cdot)\right]^{T}$ is a set of nonlinear functions that must be carefully adjusted to invert the action of $\mathbf{f}(\cdot)$, and $\mathbf{W}$ corresponds to the linear separating matrix.

\section{SEPARATION OF BANDLIMITED SIGNALS IN A PNL MODEL}

The peculiar structure of the PNL mixture, with a clear division between the linear and the nonlinear sections, suggests that one could treat each stage separately. If that is the case, after canceling the nonlinear distortion introduced by the functions $f_{i}(\cdot)$, the problem is reduced to the one of linear BSS, for which efficient tools are available. Our approach follows this idea and, in the sequel, we will discuss how it becomes possible to deal with the nonlinear stage when the sources are bandlimited signals. In our development, we also assume that the sources are mutually independent and that the number of mixtures is, at least, equal to the number of sources. In fact, these two additional assumptions are necessary if one wishes to use the standard BSS methods in the linear mixing stage.

\subsection{Compensating the nonlinear functions}

Let us assume that each source $s_{i}(t)$ is a bandlimited signal with maximum frequency given by $B_{s_{i}(t)}$. Then, given that the Fourier transform is a linear operator, the signals $z_{i}(t)$, which correspond to linear mixtures of the sources, are also bandlimited signals with maximum frequency given by $B_{z_{i}(t)}=\max \left(B_{s_{1}(t)}, \cdots, B_{s_{n}(t)}\right)$. Based on a well-known result from the nonlinear signal processing theory (see $[5,6]$ for instance), we know that if the bandlimited signal $z_{i}(t)$ is submitted to a nonlinear function $f(\cdot)$, then the spectrum of the resulting signal $x_{i}(t)=f\left(z_{i}(t)\right)$ will be wider than the spectrum of the original signal.

In order to show why ${ }^{1}$ spectral spreading takes place in nonlinear systems, let us assume that $f_{i}(\cdot)$ admits a power series expansion, i.e.,

$$
x_{i}(t)=f_{i}\left(z_{i}(t)\right)=\sum_{k=1}^{\infty} f_{i}^{(k)} z_{i}(t)^{k} .
$$

Denoting by $Z_{i}(\omega)$ the Fourier transform of $z_{i}(t)$, the Fourier transform of (3) is given by

$$
\begin{aligned}
X_{i}(\omega)= & f_{i}^{(1)} Z_{i}(\omega)+f_{i}^{(2)} Z_{i}(\omega) * Z_{i}(\omega)+ \\
& f_{i}^{(3)} Z_{i}(\omega) * Z_{i}(\omega) * Z_{i}(\omega)+\cdots,
\end{aligned}
$$

where the symbol ' $*$ ' stands for the convolution operator. A basic property of the convolution states that if $R_{1}(\omega)$ and $R_{2}(\omega)$ denote the Fourier transform of two signals bandlimited to $B_{1}$ and $B_{2}$, respectively, then $R_{1}(\omega) * R_{2}(\omega)$ is bandlimited to $B_{1}+B_{2}$ [6]. Therefore, in Eq. (4), since $Z_{i}(\omega)$ is bandlimited to $B_{z_{i}(t)}$, then $Z_{i}(\omega) * Z_{i}(\omega)$ will be bandlimited to $2 B_{z_{i}(t)}, Z_{i}(\omega) * Z_{i}(\omega) * Z_{i}(\omega)$ to $3 B_{z_{i}(t)}$, and so forth. As a consequence, it is expected that the maximum frequency of $X_{i}(\omega)$ be larger than $B_{z_{i}(t)}$.

In view of the spectral spreading phenomenon described above, the nonlinear effects introduced by $f_{i}(\cdot)$ can be counterbalanced by a function $g_{i}(\cdot)$ that gives a signal $q_{i}(t)$ bandlimited to the original bandwidth of the input signal $z_{i}(t)$. Indeed, in view of Eq. (4), this condition is satisfied when $q_{i}(t)=g_{i}\left(f_{i}\left(z_{i}(t)\right)\right)=\alpha z_{i}(t)+\beta$, where $\alpha, \beta \in \mathbb{R}$, that is, when the composition of the two functions becomes a linear function. In the sequel, we shall discuss how this idea can be put into practice.

\subsection{Implementation issues}

Since we are interested in finding a function $g_{i}\left(\cdot, \mathbf{w}_{i}\right)$, parametrized by $\mathbf{w}_{i}$, that gives a signal $q_{i}(t)$ whose energy

\footnotetext{
${ }^{1}$ We refer the reader to [6] for a more complete explication of this phenomenon.
} 
beyond the frequency $B_{z_{i}(t)}$ is as low as possible, we can formulate this task as the following minimization problem

$$
\min _{\mathbf{w}_{i}} J_{1}\left(\mathbf{w}_{i}\right)=\frac{E_{q_{i}(t)}^{f>B_{z_{i}(t)}}}{E_{q_{i}(t)}}
$$

where $E_{q_{i}(t)}$ denotes the total energy of $q_{i}(t)$ and $E_{q_{i}(t)}^{f>B_{z_{i}}(t)}$ the energy associated with the frequency components greater than $B_{z_{i}(t)}$. The purpose of the term $E_{q_{i}(t)}$ is to avoid a trivial solution where the signal $q_{i}(t)$ has null energy.

The cost function defined in (5), which is the basis of the approach developed in [6], works with the strong assumption that $B_{z_{i}(t)}$ is known in advance. As a consequence, its application is not possible in a blind source separation context, as such an information is usually not available. Yet, it would be possible to extend $J_{1}\left(\mathbf{w}_{i}\right)$ to a complete blind scenario with unknown $B_{z_{i}(t)}$. In fact, we could replace, in Eq. (5), $B_{z_{i}(t)}$ by a value $\hat{B}_{z_{i}(t)}$ that satisfies $\hat{B}_{z_{i}(t)}>B_{z_{i}(t)}$ (for instance, this can be achieved by selecting $\hat{B}_{z_{i}(t)}$ close to one $\left.{ }^{2}\right)$. In this new situation, we are thus trying to minimize the spectral spreading in the band $\left[\hat{B}_{z_{i}(t)}>B_{z_{i}(t)}, 1\right]$. Evidently, since this is only a necessary condition, there is no theoretical guarantee that such a procedure will lead to a proper compensation of $f_{i}(\cdot)$. On the other hand, this procedure usually performs well in practice, at least in noiseless situations.

When the mixtures are corrupted by noise, it turns out that the complete blind strategy described in the last paragraph may become rather suboptimal. For example, suppose that $\hat{B}_{z_{i}(t)}>>B_{z_{i}(t)}$, i.e. our guess is much more higher that the actual bandwidth of $z_{i}(t)$. Then, the criterion (5) will consider only a few high-frequency components, whereas all the information available in the band $\left[B_{z_{i}(t)}, \hat{B}_{z_{i}(t)}\right]$ will be discarded. Therefore, the resulting estimator in this case will be much less robust to noise than the estimator considering the actual value $B_{z_{i}(t)}$. This is particularly undesirable in our problem given that even a low-power noise can become significant after the nonlinear functions.

In view of the limitations associated with the blind extension of the paradigm introduced by Eq. (5), a more reasonable approach is to define a cost function in which $B_{z_{i}(t)}$ is also considered as an unknown parameter. For instance, this can be done through the following minimization problem

$$
\min _{\mathbf{w}, \hat{B}_{z_{i}(t)}} J_{2}\left(\mathbf{w}, \hat{B}_{z_{i}(t)}\right)=\frac{E_{q_{i}(t)}^{f>\hat{B}_{z_{i}(t)}}}{E_{q_{i}(t)}^{f>\hat{B}_{z_{i}(t)}-b}},
$$

where the parameter $b$ lies inside $] 0,1[$ and should be assigned in advance. Later, we will discuss how this can be done.

\footnotetext{
${ }^{2}$ In this work, we consider that the signals are already in a discrete-time representation. Given that, we always refer to the normalized frequency, where $B=1$ corresponds, in the analog domain, to $F_{s} / 2$, where $F_{s}$ is the sampling frequency.
}

It is interesting to note that the cost function (6) attains a small value whenever there is a great variation between the energies of $q_{i}(t)$ in the bands $\left[\hat{B}_{z_{i}(t)}, 1\right]$ and $\left[\hat{B}_{z_{i}(t)}-b, 1\right]$. This situation is expected for the desired solution to our problem, i.e., when $\left(\mathbf{w}=\mathbf{w}_{d}, \hat{B}_{z_{i}(t)}=B_{z_{i}(t)}\right)$, where $\mathbf{w}_{d}$ represents the parameters that provide the inversion of $f_{i}(\cdot)$. Indeed, when $g_{i} \circ f_{i}$ is almost linear, one expects a very low energy $E_{q_{i}(t)}^{f>B_{z_{i}(t)}}$, given that the high-frequency components introduced by $f_{i}(\cdot)$ into $q_{i}(t)$ are quite reduced. Concerning the term $E_{q_{i}(t)}^{f>B_{z_{i}(t)}-b}$, it includes the energy $E_{q_{i}(t)}^{f>B_{z_{i}(t)}}$ but also the energy $E_{q_{i}(t)}^{B_{z_{i}(t)}-b<f<B_{z_{i}(t)}}$. This last term lies within the bandwidth of $z_{i}(t)$, and, as a consequence, is expected to be much larger than $E_{q_{i}(t)}^{f>B_{z_{i}(t)}}$, which thus explains why $J_{2}\left(\mathbf{w}_{d}, B_{z_{i}(t)}\right)$ is expected to be quite small.

It is worth noting that significant variations between $E_{q_{i}(t)}^{f>\hat{B}_{z_{i}(t)}}$ and $E_{q_{i}(t)}^{f>\hat{B}_{z_{i}(t)}-b}$ may also happen if the spectrum of $q_{i}(t)$ presents energy variations as, for instance, an attenuated band. As a consequence, the cost function (6) tends to present local modes around the points $\hat{B}_{z_{i}(t)}$ where these variations occur. A practical consequence of this observation regards the definition of the optimization algorithm: as $J_{2}\left(\mathbf{w}, \hat{B}_{z_{i}(t)}\right)$ may be multimodal, the application of methods based only on local search mechanisms, such as the pure gradient-based techniques, is not recommended since they may converge to local minima.

Another important practical point regards the role of $b$ in $J_{2}\left(\mathbf{w}, \hat{B}_{z_{i}(t)}\right)$. This parameter acts as a sort of frequency resolution. For example, if the input signal is periodic (pure harmonics), then $b$ should be small as the energy variations are high concentrated in the spectrum. Conversely, for aperiodic signals, the energy is less concentrated in the spectrum and, thus, a greater value for $b$ must be defined. In practice, we observed that a good rule of thumb is to select $b=0.01$ for periodic signals and $b=0.1$ for aperiodic signals.

\subsubsection{Summary of the final algorithm for PNL source sepa- ration}

Having discussed how the nonlinear inversion of $f_{i}(\cdot)$ can be carried out, the complete algorithm for PNL source separation can be summarized as follows:

1. First stage. For each mixture $x_{i}(t)$, find $g_{i}\left(x_{i}(t), \mathbf{w}_{i}\right)$ by minimizing the cost function $J_{2}\left(\mathbf{w}_{i}, \hat{B}_{z_{i}(t)}\right)$ (Eq. (6));

2. Second stage. The estimated sources $y_{i}(t)$ are obtained by applying a linear source separation or extraction method to the signals $q_{i}(t)=g_{i}\left(x_{i}(t)\right)$.

As it was discussed before, due to the existence of local optima in $J_{2}\left(\mathbf{w}_{i}, \hat{B}_{z_{i}(t)}\right)$, care must be taken in the definition of an optimization method adopted in the first stage. In this work, we adopted the opt-aiNet algorithm [7], an evolutionary method that has been proved to be very efficient in solving 
multimodal optimization problems. As explained in [7], the opt-aiNet requires exclusively the evaluation of the cost function to be optimized. Therefore, in view of Eq. (6), it becomes necessary to evaluate the energy of $q_{i}(t)$ in a given frequency band. This can be done by calculating the frequency content of $q_{i}(t)$ via, for instance, the discrete cosine transform $(\mathrm{DCT})^{3}$. Then, the energy is given by the Euclidean norm of the DCT coefficients associated with the desired band.

\section{RESULTS}

We present in this section a set of simulations performed in order to check the effectiveness of the procedure proposed in Section 3.2.1. Firstly, we focus on the initial stage, which concerns the nonlinear compensation of a PNL model. After that, we present an example that shows the usefulness of our proposal in a complete PNL source separation framework.

\subsection{Inversion of the nonlinear stage}

In order to illustrate the applicability of the cost function (6), let us consider an example of PNL mixing model with 2 sources and 2 mixtures. The two sources, whose bandwidths are given by $B_{s_{1}(t)}=0.2$ and $B_{s_{2}(t)}=0.5$, were obtained from low-pass FIR filters (100 taps) driven by white Gaussian noise. The linear part of the PNL mixing system is given by the matrix $\mathbf{A}=[10.5 ; 0.61]$. Concerning the nonlinear component-wise functions $f_{i}(\cdot)$, our analysis encompassed two representative cases: the Nicolsky-Eisenman (NE) model and the situation where the inverting functions $g_{i}(\cdot)$ are polynomials. We will discuss these models in the sequel.

\subsubsection{Nicolsky-Eisenman model}

One of the applications of PNL models is related to the use of ion-selective electrodes (ISEs) array in the problem of estimating the concentrations of several ions in aqueous solution [8]. Typically, an ISE lacks total selectivity, that is, it may respond to a given target but also to other interfering ions within the solution. As a result of this phenomenon, the outputs of an ISE array become mixed versions of a set of source signals, i.e., the concentrations of each ion within the solution. This mixing process can be modeled according to the classical formalism of the Nicolsky-Eisenman (NE) equation, which states that, if the ions under analysis have the same valences, which is indeed very common in practice, then the response of each ISE within the array is given by

$$
x_{i}(t)=e_{i}+d_{i} \log \left(\sum_{j=1}^{n_{s}} a_{i j} s_{j}(t)\right),
$$

\footnotetext{
${ }^{3}$ We could equally adopt the discrete Fourier transform (DFT). However, the DCT has the advantage of being a real-valued transform. Furthermore, we checked through preliminary simulations that the DCT gives better results for aperiodic signals.
}

where $e_{i}, d_{i}$ and $a_{i j}$ denote unknown mixing coefficients.

From Eq. (7), it becomes clear that the NE model is an example of PNL model. The inversion of the nonlinear stage in this case can be achieved by means of the following functions

$$
q_{i}(t)=g_{i}\left(x_{i}(t), \hat{d}_{i}\right)=\exp \left(\frac{x_{i}(t)}{\hat{d}_{i}}\right)=z_{i}(t)^{\frac{d_{i}}{\hat{d}_{i}}} .
$$

If, and only if, $\hat{d}_{i}=d_{i}$, the composition $g_{i} \circ f_{i}$ is linear and, thus, this situation corresponds to the desired solution.

Since the function $g_{i}\left(x_{i}(t), \hat{d}_{i}\right)$ is parametrized by just one parameter, it becomes possible to visualize the shape of the cost function (6) in this case. For example, in a noiseless situation in which $d_{1}=0.059$ and $d_{2}=0.040$, the cost functions for both $g_{1}(\cdot)$ and $g_{2}(\cdot)$ are shown in Fig. 2. Note that the values $\hat{d}_{i}$ that minimize $J_{2}\left(\hat{d}_{i}, \hat{B}_{z_{i}(t)}\right)$ coincide with the actual values of $d_{i}$. Moreover, the proposed criterion is minimized for both cases when $\hat{B}_{z_{i}(t)}=0.54$, which is close to the bandwidth of the linear mixtures $\left(B_{z_{i}(t)}=0.5\right)$.

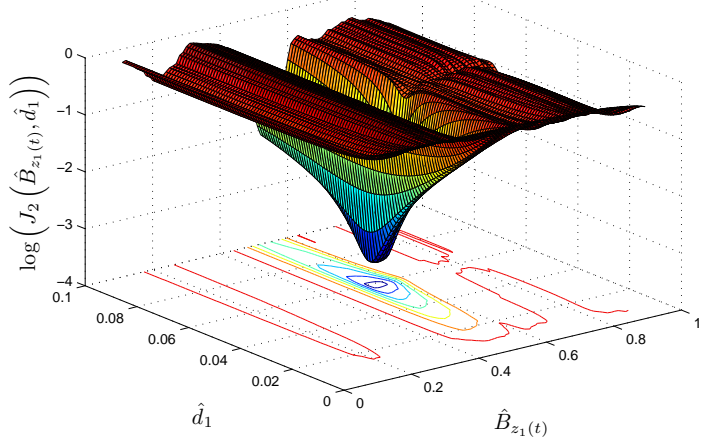

(a) $J_{2}\left(\hat{d}_{1}, \hat{B}_{z_{1}(t)}\right)$.



(b) $J_{2}\left(\hat{d}_{2}, \hat{B}_{z_{2}(t)}\right)$.

Fig. 2. Cost functions $J_{2}\left(\hat{d}_{i}, \hat{B}_{z_{i}(t)}\right)$ for the NE model.

As discussed in Sec. 3.2, there may exist locally optimal models if there are energy variations in the spectrum of $z_{i}(t)$. This phenomenon is clear in Fig. 2(a) where one can observe a local mode around the frequency $\hat{B}_{z_{i}(t)}=0.2$. In this 
Table 1. Effects of noise on the estimation of $d_{2}$.

\begin{tabular}{cccc}
\hline & $\hat{d}_{2}(S N R=18 \mathrm{~dB})$ & $\hat{d}_{2}(S N R=20 \mathrm{~dB})$ & $\hat{d}_{2}$ (noise free) \\
\hline $\begin{array}{c}J_{1}\left(\hat{d}_{2}\right) \\
\text { (semi-blind) }\end{array}$ & 0.0515 & 0.0455 & 0.0395 \\
\hline$J_{1}\left(\hat{d}_{2}, \hat{B}_{z_{2}(t)}\right)$ & 0.3336 & 0.1085 & 0.0398 \\
\hline $\begin{array}{c}\text { (blind) } \\
J_{2}\left(\hat{d}_{2}, \hat{B}_{z_{2}(t)}\right)\end{array}$ & 0.0490 & 0.0457 & 0.0396 \\
\hline
\end{tabular}

case, the energy variation around this frequency takes place because $z_{i}(t)$ is a linear combination of two bandlimited signals, one of them having a bandwidth equal to $B_{s_{1}(t)}=0.2$.

A relevant point in nonlinear systems like the NE model regards the effect of the noise. Indeed, even a low-energy noise can be problematic in this case. For example, let us assume that the observations are given by $x_{i}(t)+n_{i}(t)$, where $n_{i}(t)$ denotes a zero-mean additive white Gaussian (AWG) noise. Due to the exponential function $g_{i}(\cdot)$, one has $z_{i}(t) \propto$ $x_{i}(t)^{d_{i} / \hat{d}_{i}} \exp \left(n_{i}(t)\right)$, i.e. $z_{i}(t)$ is corrupted by a multiplicative noise that follows a log-normal distribution. This may result in a noise amplification, specially when the input values of $g_{i}(\cdot)$ are high.

We conducted a set of simulations to investigate the effects of the noise on the estimations of $\hat{d}_{2}$ obtained by the following approaches: 1) the estimator associated with $\left.J_{2}\left(\hat{d}_{2}, \hat{B}_{z_{2}(t)}\right), 2\right)$ the estimator associated with $J_{1}\left(\hat{d}_{Z}\right)$ assuming the knowledge of the bandwidth $B_{z_{2}(t)}$ (semi-blind case), 3) the same cost function $J_{1}\left(\hat{d}_{2}\right)$ but now in a complete blind situation, in which $B_{z_{2}(t)}$ is defined beforehand (we set $\hat{B}_{z_{2}(t)}=0.8$ ). In Tab. 1 , which represents the average of 100 experiments, one can note that, in a noiseless scenario, the three estimators give values closer to the actual one $\left(d_{2}=0.040\right)$. When there is noise, the blind version of $J_{1}\left(\hat{d}_{2}\right)$ gives poor estimations for $d_{2}$, whereas $J_{1}\left(\hat{d}_{2}\right)$ and $J_{2}\left(\hat{d}_{2}, \hat{B}_{z_{2}(t)}\right)$ still work properly. However, it worth remembering that, while $J_{1}\left(\hat{d}_{2}\right)$ assumes the knowledge of the actual bandwidth of the input signal, $J_{2}\left(\hat{d}_{2}, \hat{B}_{z_{2}(t)}\right)$ operates in a completely blind fashion.

\subsubsection{Polynomial model}

In a second situation, we considered that the nonlinear mixing functions are given by $f_{i}\left(z_{i}(t)\right)=\sqrt[3]{z_{i}(t)}$. To compensate them, we make use of polynomial functions given by:

$$
q_{i}(t)=g_{i}\left(x_{i}(t), \mathbf{w}_{i}\right)=a_{i} x_{i}^{5}(t)+b_{i} x_{i}^{3}(t)+c_{i} x_{i}(t) .
$$

The expected solution (in a noiseless case) is thus given by $a_{i}=c_{i}=0$ and $b_{i}=\delta$, where $\delta \in \mathbb{R}$. Note that in this case one can fix, without loss of generality, $b_{i}=1$.

In order to check if $J_{2}\left(\mathbf{w}, \hat{B}_{z_{i}(t)}\right)$ succeeds in discriminating the desired solution, we plot in Fig. 3 the shapes of this cost function for the two mixtures of our example. Since this function depends on three parameters $\left(a_{i}, c_{i}\right.$ and $\left.\hat{B}_{z_{i}(t)}\right)$, we had to fix $\hat{B}_{z_{i}(t)}$ to the value that minimizes $J_{2}\left(\mathbf{w}, \hat{B}_{z_{i}(t)}\right)$
$\left(\hat{B}_{z_{i}(t)}=0.52\right.$ in this case). By looking at the resulting shapes, one can note that the proposed cost function is indeed minimized by the expected solutions of our problem.

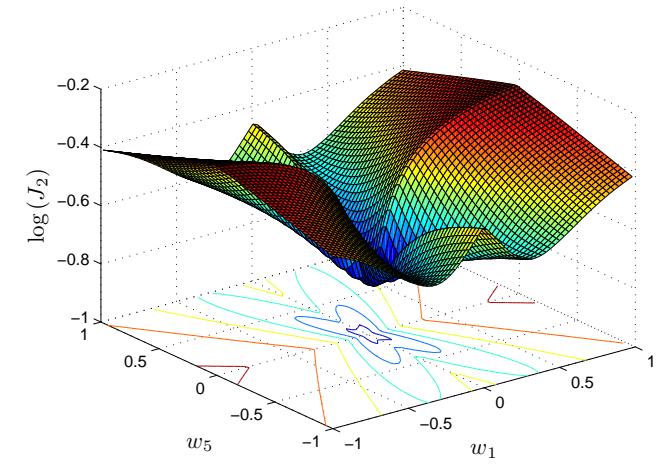

(a) $J_{2}\left(\mathbf{w}_{1}, \hat{B}_{z_{1}(t)}\right)$.

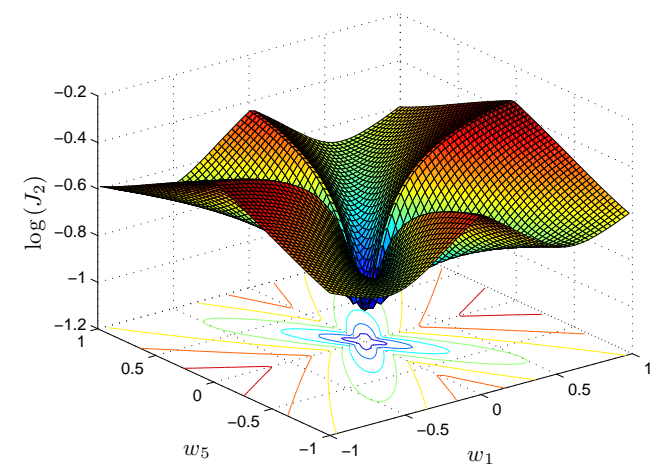

(b) $J_{2}\left(\mathbf{w}_{2}, \hat{B}_{z_{2}(t)}\right)$.

Fig. 3. Cost functions $J_{2}\left(\mathbf{w}_{i}, \hat{B}_{z_{i}(t)}\right)$ for the polynomial model.

\subsection{Example of PNL source separation}

We now present an example where the complete procedure, described in Section 3.2.1, is applied to a PNL mixture with 3 sources and 3 sensors. The first source is a sine wave of frequency $B_{s_{1}(t)}=0.01$, while the two others are aperiodic signals with bandwidth $B_{s_{2}(t)}=0.5$ and $B_{s_{3}(t)}=0.8$. The linear part of the mixing system is given by the matrix

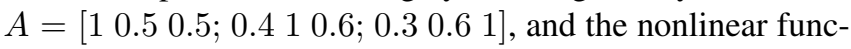
tions followed the NE model, i.e., $f_{i}\left(x_{i}\right)=d_{i} \log \left(x_{i}\right)$ where $d_{1}=0.050, d_{2}=0.060$ and $d_{3}=0.045$. The number of available samples in this situation was 1000, and an AWG noise of $S N R=20 \mathrm{~dB}$ was defined in each sensor.

The application of the proposed method provided the following estimations: $\hat{d}_{1}=0.048, \hat{d}_{2}=0.070$ and $\hat{d}_{3}=0.044$. As can be seen in Fig. 4, the resulting mappings between $z_{i}(t)$ and $q_{i}(t)$ are close to linear functions, which indicates that 
the task of inverting the nonlinearities was satisfactorily accomplished. Yet, it is clear in this figure the noise amplification phenomenon discussed in Section 4.1.1, and also that the noise effect grows as the input value grows.

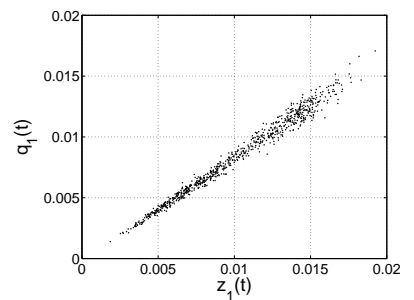

(a) $q_{1}(t)=g_{1}\left(z_{1}(t)\right)$.

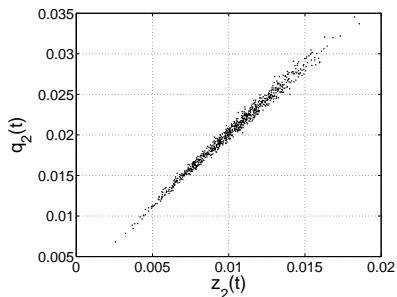

(b) $q_{2}(t)=g_{2}\left(z_{2}(t)\right)$.

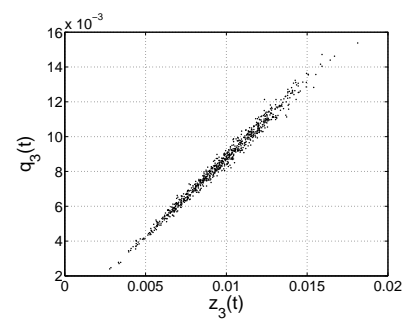

(c) $q_{3}(t)=g_{3}\left(z_{3}(t)\right)$.

Fig. 4. Mappings between $z_{i}(t)$ and $q_{i}(t)$ for each channel.

Having dealt with the nonlinear functions, we applied the SOBI algorithm [9] on the signals $q_{i}(t)$. This method provided good estimations of the actual sources. Indeed, the obtained performance indices ${ }^{4}$ were $S I R_{1}=14.94 \mathrm{~dB}$, $S I R_{2}=10.62 d B$ and $S I R_{3}=12.02 d B$. In Fig. 5, we show the source $s_{1}(t)$ (the sine wave) together with its corresponding estimation $y_{1}(t)$. Note that, due to effect of noise amplification mentioned before, the estimation error is more evident when the signal attains high values.

\section{CONCLUSIONS}

We proposed a novel source separation method for postnonlinear mixtures. Based on the assumption that the sources are bandlimited signals, we could obtain a two-stage solution, where the compensation of the nonlinear section of the PNL was achieved by functions adjusted with the aim of canceling the high-order frequency terms introduced by the nonlinear distortions. Then, the linear stage could be estimated through standard BSS methods. To implement this idea, we proposed a cost function that can be applied even in noisy scenarios. The simulation results attested the viability of the proposed approach. Future works include the extension of our method to the case of broadband signals.

\footnotetext{
${ }^{4} \mathrm{We}$ adopted the following performance index: $S I R_{i}=$ $10 \log \left(\frac{E\left\{\hat{s}_{i}(t)^{2}\right\}}{E\left\{\left(\hat{s}_{i}(t)-\hat{y}_{i}(t)\right)^{2}\right\}}\right)$, where $\hat{y}_{i}(t)$ and $\hat{s}_{i}(t)$ denote, respectively, the retrieved signal and the actual source after mean and variance normalization.
}

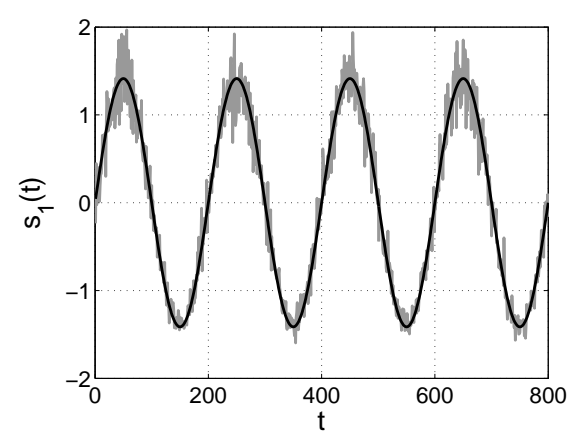

Fig. 5. Actual source $s_{1}(t)$ (black) and estimated source $y_{1}(t)$ (gray).

\section{REFERENCES}

[1] C. Jutten and J. Karhunen, "Advances in blind source separation (BSS) and independent component analysis (ICA) for nonlinear mixtures," International Journal of Neural Systems, vol. 14, pp. 267-292, 2004.

[2] A. Taleb and C. Jutten, "Source separation in postnonlinear mixtures," IEEE Trans. on Signal Processing, vol. 47, no. 10, pp. 2807-2820, 1999.

[3] J. Solé-Casals, M. Babaie-Zadeh, C. Jutten, and D.-T. Pham, "Improving algorithm speed in pnl mixture separation and wiener system inversion," in Proceedings of the ICA 2003, 2003.

[4] M. Babaie-Zadeh, C. Jutten, and K. Nayebi, "A geometric approach for separating post non-linear mixtures," in Proceedings of the EUSIPCO 2002, Toulouse, France, 2002, pp. 11-14.

[5] E. Masry, "The recovery of distorted band-limited stochastic processes," IEEE Trans. on Information Theory, vol. 19, no. 4, pp. 398-403, 1973.

[6] K. Dogançay, "Blind compensation of nonlinear distortion for bandlimited signals," IEEE Trans. on Circuits and Systems I, vol. 52, no. 9, pp. 1872-1882, 2005.

[7] L. N. de Castro and J. Timmis, "An artificial immune network for multimodal function optimization," in Proc. of ICEC'02, Honolulu, Hawaii, 2002, pp. 699-674.

[8] L. T. Duarte, C. Jutten, and S. Moussaoui, "Ion-selective electrode array based on a Bayesian nonlinear source separation method," in Proceedings of the ICA 2009, 2009.

[9] A. Belouchrani, K. Abed-Meraim, J.-F. Cardoso, and E. Moulines, "A blind source separation technique using second-order statistics," IEEE Trans. on Signal Processing, vol. 45, no. 2, pp. 434-444, 1997. 